\title{
Universal Transfer of 2D Materials Grown on Au Substrate Using Sulfur Intercalation
}

\author{
Received 8 December, 2020; revised 21 January, 2021; accepted 26 January, 2021
}

\author{
Soo Ho Choi ${ }^{a}$, Ji Hoon Choi ${ }^{b}$, Chang Seok Oh ${ }^{b}$, Gyeongtak Han ${ }^{b}$, Hu Young Jeong ${ }^{c}$, Young-Min Kim ${ }^{a, b}$, Soo \\ Min Kim $^{d, *}$, and Ki Kang Kim ${ }^{a, b, *}$ \\ aCenter for Integrated Nanostructure Physics (CINAP), Institute for Basic Science (IBS), Sungkyunkwan University, \\ Suwon 16419, Republic of Korea \\ bDepartment of Energy Science, Sungkyunkwan University, Suwon 16419, Republic of Korea \\ ¿UNIST Central Research Facilities and Department of Materials Science and Engineering, UNIST, UIsan 44919, Re- \\ public of Korea \\ dDepartment of Chemistry, Sookmyoung Women's University, Seoul 14072, Republic of Korea
}

\section{*Corresponding author E-mail: kikangkim@skku.edu, soominkim@sookmyung.ac.kr}

\begin{abstract}
Herein, we report on a novel method for transferring two-dimensional (2D) materials grown on Au substrates using sulfur intercalation between the 2D materials and the Au surfaces. The strong nature of the $\mathrm{S}-\mathrm{Au}$ bond allows intercalation of sulfur atoms into their interface, under a sulfurrich atmosphere, at $600{ }^{\circ} \mathrm{C}$. The relaxed interfacial interaction achieved via intercalation is carefully confirmed by recovering phonon mode and work function of tungsten disulfide $\left(\mathrm{WS}_{2}\right)$ in Raman spectra and Kelvin probe force microscopy, and, more importantly, by observing the expansion of the interfacial distance, from 0.24 to $0.44 \mathrm{~nm}$, using cross-sectional transmission electron microscopy. The released interactions facilitate delamination of $\mathrm{WS}_{2}$ from the Au surface, using an electrochemical bubbling method. The resultant Au foil then is reused for repeated $\mathrm{WS}_{2}$ growth. The successful transfer of other $2 \mathrm{D}$ materials, including molybdenum disulfide and hexagonal boron nitride, is also demonstrated. Our strategy advances the use of Au substrates for growing wafer-scale 2D monolayers.
\end{abstract}

Keywords: Two-dimensional materials, Transition metal dichalcogenides, Chemical vapor deposition, Gold, Intercalation

\section{Introduction}

Interest in two-dimensional (2D), van der Waals (vdW) layered materials, including graphene (Gr), transition metal dichalcogenides (TMDC), and hexagonal boron nitride (hBN), has recently increased, owing to their unusual physical and chemical properties [1-6]. To utilize those emerging materials into industrial applications, wafer-scaled growth of 2D materials is highly desired. Among various growth techniques including molecular beam epitaxy, atomic layer deposition, puls -ed layer deposition, and chemical vapor deposition (CVD) [7-10], CVD method has been mostly employed, due to its several advantages compared to other growth techniques, which include mass production, cost-effectiveness, and low temperature operation.

The substrate is most important key ingredient for the CVD growth of $2 \mathrm{D}$ materials to control morphology, orientation, and growth modes [10-22]. While chemically stable insulating substrates such as $\mathrm{SiO}_{2}$, $\mathrm{Al}_{2} \mathrm{O}_{3}$, glass, and quartz enable to grow the various poly-crystalline TMDCs film, the lack of catalytic activity impedes the synthesis of $\mathrm{Gr}$ and $\mathrm{hBN}$ film. On the other hand, the catalytic metal substrates such as $\mathrm{Cu}, \mathrm{Ni}$, and $\mathrm{Fe}$ have been widely exploited for the growth of $\mathrm{Gr}$ and $\mathrm{hBN}$, but the high chemical reactivity with chalcogens and transition metals to form alloy and compound, prohibits to use as the growth substrate for TMDCs. To date, only Au metal can serve as universal growth substrate for most of $2 \mathrm{D}$ materials owing to possessing the unique characteristics of high chemical inertness (that is, no alloy and compound formation), scarce $\mathrm{B}, \mathrm{N}, \mathrm{S}$, and transition metal solubilities in bulk $\mathrm{Au}$, and its ability to act as a catalyst for activating precursors.
Recently, wafer-scale, single-crystal (SC) 2D films such as hBN, $\mathrm{WS}_{2}, \mathrm{MoS}_{2}, \mathrm{WSe}_{2}, \mathrm{MoSe}_{2} / \mathrm{WSe}_{2}$ lateral heterostructure, and $\mathrm{W}_{1-\mathrm{x}} \mathrm{Mo}_{\mathrm{x}}$ $-\mathrm{S}_{2}$ alloy have been successfully synthesized on Au foil, using the selfcollimation and epitaxial growth methods $[16,20]$. When using SC 2D materials in practical device applications, it inevitably becomes necessary to transfer $2 \mathrm{D}$ film onto other substrates. CVD-grown $2 \mathrm{D}$ materials are typically transferred after wet-etching the growth substrate. For example, $\mathrm{Cu}$ foils used for graphene growth have typically been etched away in etchant solution [21], while for more costly Au foils, non-destructive methods, such as electrochemical bubble transfers, have been more popular than wet-etching process. A few studies have reported transferring 2D material grown on Au foils via electrochemical bubbling $[16,18]$, although it was found that the strong Au-S interaction reduced the efficiency with which $2 \mathrm{D}$ materials could be detached from the Au surface during electrochemical bubbling, leading to poor transfer yields [18]. Despite this, it appears that relaxing this strong interfacial interaction to attain a higher transfer yield has not been studied.

Here, we report a universal transfer method for 2D material grown on $\mathrm{Au}$ foil, using S-intercalation into the interface between the 2D material and the Au surface. The S-intercalation was carried out by annealing in an S-rich atmosphere, at $600{ }^{\circ} \mathrm{C}$. The interfacial distance between $\mathrm{WS}_{2}$ and the Au surface had increased, from 0.24 to $0.44 \mathrm{~nm}$, reducing the interfacial interaction. Such relaxed interaction restored the $\mathrm{WS}_{2}$ electronic structure toward its undoped state, and, more importantly, allowed easy $\mathrm{WS}_{2}$ film delamination from the Au surface, using electrochemical bubbling. Eventually, the cm-scale $\mathrm{WS}_{2}$ film

This is an Open Access article distributed under the terms of the Creative Commons Attribution Non-Commercial License (http://creativecommons.org/licenses/by-nc/3.0) which permits unrestricted non-commercial use, distribution, and reproduction in any medium, provided the original work is properly cited. 
(a)

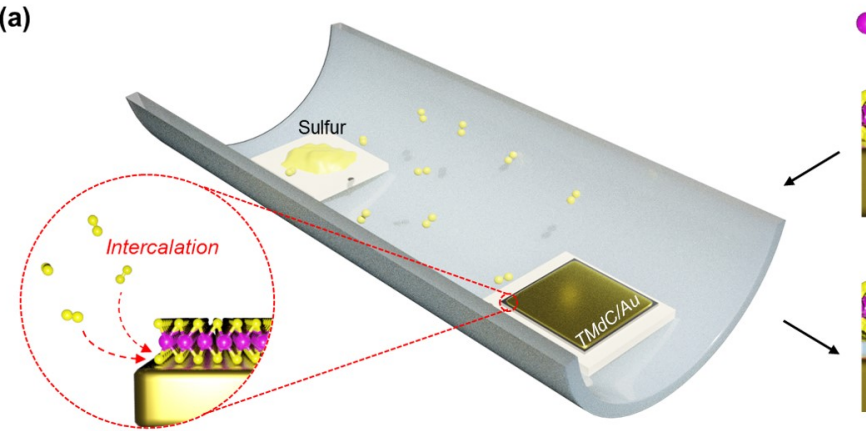

: Transition metal $\quad \circ$ : Chalcogen

As-grown TMdC

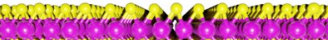

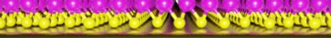

$\mathrm{Au}$

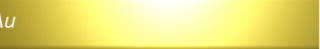

(b)

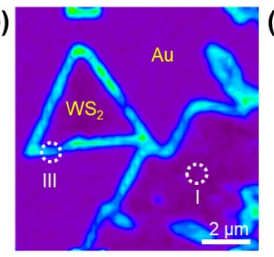

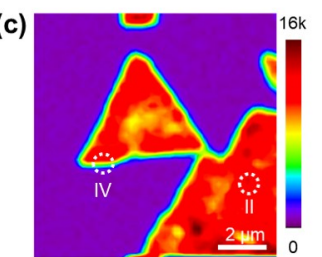

(d)

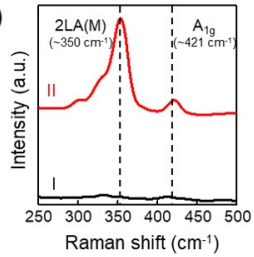

(e)

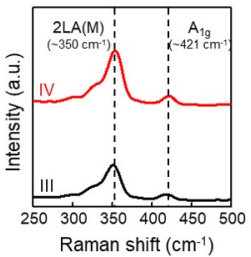

Figure 1. (a) Schematic of the intercalation process. The S atoms are intercalated between the TMdC and Au layers, under an S-rich atmosphere, at $600{ }^{\circ} \mathrm{C}$. Confocal Raman mapping images of (b) as-grown and (c) intercalated WS $_{2}$ grains, for $2 \mathrm{LA}(\mathrm{M})$ mode intensity. (d) and (e) Representative Raman spectra extracted from regions I-IV in (b) and (c).

was successfully transferred onto a $\mathrm{SiO}_{2}$ substrate. Importantly, we have also shown that the Au foils could be reused for repeated $\mathrm{WS}_{2}$ growth cycles, and have demonstrated the effective transfers of monolayer $\mathrm{MoS}_{2}$ and hBN grown on Au substrates.

\section{Experimental details}

\subsection{Substrate preparation}

$1 \times 1 \mathrm{~cm}^{2}$, high-purity Au foils (0.2 mm thick, $99.99 \%$, iNexus Inc.) were cleaned using ultra-sonication in acetone and isopropyl alcohol respectively, for $30 \mathrm{~s}$. The surface impurities on Au substrates were then removed by dipping into Au etchant (GE-8111, Transene), for 10 min. The etchant residue was rinsed away using deionized (DI) water and the $\mathrm{Au}$ foils were then further annealed, at $1000^{\circ} \mathrm{C}$ for $1 \mathrm{~h}$, under $\mathrm{Ar}$ and $\mathrm{H}_{2}$ atmospheres with flow rates of 1000 and $50 \mathrm{sccm}$, respectively. To obtain smooth surfaces, the Au foils were melted onto a W foil (0.1-mm thick, $99.95 \%$, Alfa Aesar), at $1080{ }^{\circ} \mathrm{C}$ for $20 \mathrm{~min}$, under the same conditions. All the experiments in this work were conducted at atmospheric pressure.

\subsection{Growing TMDC monolayers}

$\mathrm{WS}_{2}$ monolayer growth was carried out through sulfurization of tungsten precursor-coated Au substrates [16]. The W precursor was prepared by dissolving $2 \mathrm{wt} \%$ sodium tungstate dihydrate in acetylacetone. The prepared solution was spun onto an Au substrate, at 2500 $\mathrm{rpm}$ for $60 \mathrm{~s}$, and an ammonium sulfide $\left[\left(\mathrm{NH}_{4}\right)_{2} \mathrm{~S}\right]$ solution, acting as a sulfur precursor, was supplied, using a bubbler system [22]. The precursor-coated Au substrate was then loaded into a 2-inch quartz tube-which had been purged for 15 min using high-purity (99.9999 $\%)$ Ar, with a flow rate of $350 \mathrm{sccm}$ - and placed into a furnace. The temperature of the furnace containing the quartz tube was increased to $800{ }^{\circ} \mathrm{C}$, over $10 \mathrm{~min}$, and was then maintained at this temperature for $15 \mathrm{~min}$, for $\mathrm{WS}_{2}$ growth. This growth took place under an $\mathrm{H}_{2}$ and $\left(\mathrm{NH}_{4}\right)_{2} \mathrm{~S}$ atmosphere, supplied at flow rates of 5 and $20 \mathrm{sccm}$, respectively. After the growth process, the quartz tube was naturally cooled to room temperature. Monolayer $\mathrm{MoS}_{2}$ flakes were grown under conditions similar to those applied for $\mathrm{WS}_{2}$ growth, except that sodium molybdate was used as the Mo precursor.

\subsection{Growing hBN monolayers}

The hBN monolayer growth method was described in our previous report [16]. Briefly, Au foil stacked on $\mathrm{W}$ foil was mounted into a 1inch quartz tube in a furnace. The temperature was elevated to 1100 ${ }^{\circ} \mathrm{C}$, under $\mathrm{Ar}$ and $\mathrm{H}_{2}$ with flow rates of 500 and $40 \mathrm{sccm}$, to establish liquid $\mathrm{Au}$ on the $\mathrm{W}$ foil, and then borazine was supplied (as the hBN precursor) for $10 \mathrm{~min}$, at the flow rate of $0.4 \mathrm{sccm}$. After hBN grains had been grown, the furnace was rapidly cooled to room temperature.

\subsection{S-intercalation}

A two-zone furnace system was used for the sulfur intercalation process. The upstream and downstream zones were used to vaporize sulfur powder, and to provide the thermal energy for sulfur intercalation, respectively. Then, $1 \mathrm{~g}$ of sulfur powder, contained in a quartz boat, and as-grown $2 \mathrm{D}$ materials on Au substrate, were loaded into the center of each zone. To avoid any damage to the 2D materials during S-intercalation, the quartz tube was completely purged initially, by supplying Ar gas with a flow rate of $500 \mathrm{sccm}$, for $30 \mathrm{~min}$. The temperatures in the two zones were increased to 340 and $600{ }^{\circ} \mathrm{C}$ for 15 $\mathrm{min}$, and then maintained at these levels for $30 \mathrm{~min}$. After finishing S-intercalation, the temperatures were allowed to lower naturally, to room temperature. The entire process was conducted under Ar and $\mathrm{H}_{2}$ atmospheres, at flow rates of 300 and $10 \mathrm{sccm}$, respectively, and at atmospheric pressure.

\subsection{Electrochemical Transfer}

The poly(methyl methacrylate) (A9 PMMA, MicroChem) layer was spun as the supporting layer onto the $2 \mathrm{D}$ material, at $4000 \mathrm{rpm}$ for 60 $\mathrm{s}$, and then dried in a $200{ }^{\circ} \mathrm{C}$ oven for $5 \mathrm{~min}$. For an electrochemical transfer, the sample and $\mathrm{Pt}$ foil were connected to cathode and anode, respectively, in a power supply [20]. The PMMA/2D material layer was delaminated from the Au surface using generated $\mathrm{H}_{2}$ bubbles under an applied voltage of 3-10 V, in $1 \mathrm{M} \mathrm{NaOH}$ electrolyte. $\mathrm{NaOH}$ residues underneath the PMMA/2D material layer were removed by floating on DI water several times, before, the PMMA/2D material layer was transferred onto a $\mathrm{SiO}_{2} / \mathrm{Si}$ substrate. To reuse $\mathrm{Au}$ substrates, they were cleaned-by dipping in piranha solution for 3-5 h, $10 \%$ 

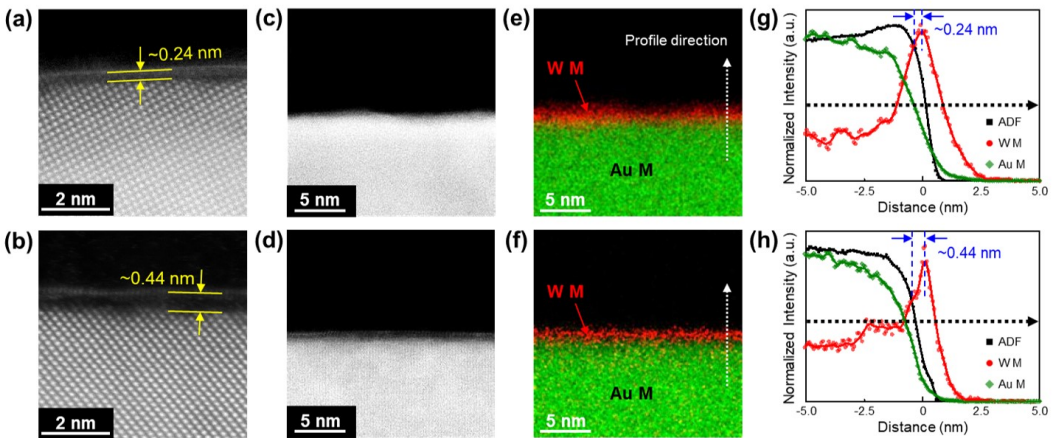

Figure 2. Cross-sectional ADF-STEM images of $\mathrm{WS}_{2}$ on Au foil (a) and (c) before, and (b) and (d) after S-intercalation, with (e) and ( $\mathrm{f}$ ) showing corresponding EDX element mapping images. $(g)$ and (h) EDX intensity profiles along the white-dashed arrows in (e) and ( $f$ ), respectively.

(a)

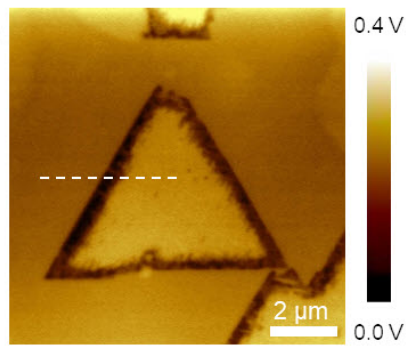

(c)

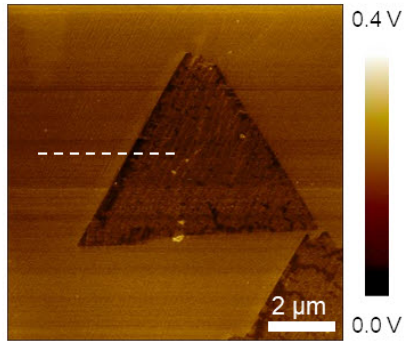

(b)

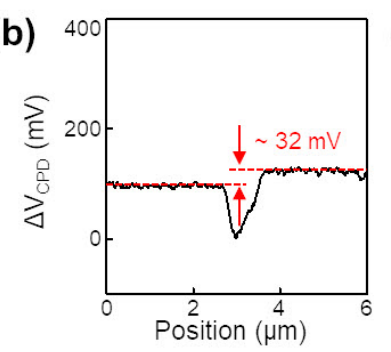

(d)

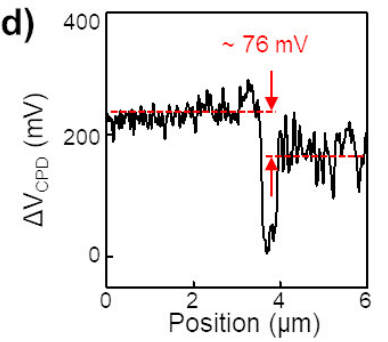

(e) As-grown WS $S_{2}$ on $A u$

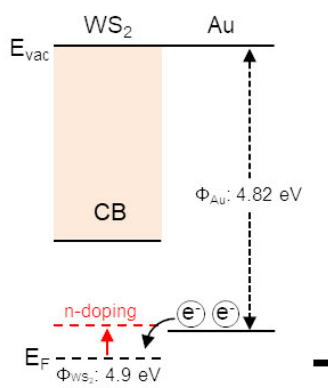

VB
S intercalated $\mathrm{WS}_{2}$ on $\mathrm{Au}$

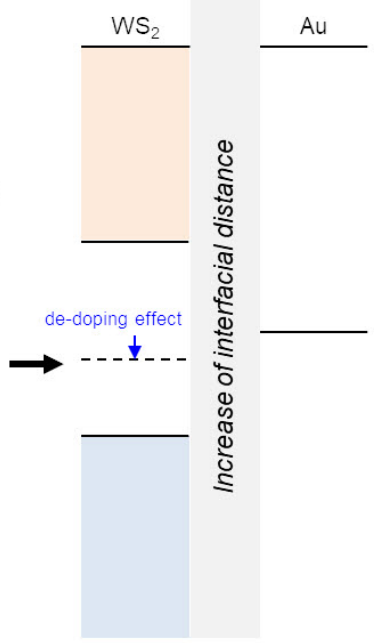

Figure 3. KPFM potential images of (a) as-grown and (c) S-intercalated WS2. (b) and (d) show KPFM potential profiles along the white-dashed lines seen in (a) and (c), respectively. (e) Energy band diagrams for $\mathrm{WS}_{2}$ and Au substrate, before and after S-intercalation.

nitric acid for $30 \mathrm{~min}$, and buffer oxide etchant for $30 \mathrm{~min}-$ prior to being reused.

\subsection{Characterization}

Overall morphologies of the TMDC and hBN monolayers on $\mathrm{Au}$ foil were characterized by field emission scanning electron microscopy (FE-SEM, JSM-7100F, JEOL) and optical microscopy (Eclipse LV150, Nikon). The cross-sectional structures and interlayer distance between $\mathrm{WS}_{2}$ and Au surface were analyzed using an aberration-corrected scanning transmission electron microscopy (STEM, JEM-ARM 200CF, JEOL), on the samples prepared by a focused ion beam (FIB, FEI Helios NanoLab 450) milling and lift-off process. Modulations of the interfacial interaction and the electronic structure by the S-intercalation were confirmed using Raman spectroscopy with a laser wavelength of $532 \mathrm{~nm}$ (XperRAM 100, Nanobase), and a Kelvin probe force microscope (KPFM, N8-NEOS, Bruker), equipped with Pt-coated atomic force microscopy (AFM) probes (Multi-75E, Budget Sensors).

\section{Results and discussion}

To intercalated $\mathrm{S}$ atoms, the annealing of as-grown $\mathrm{WS}_{2}$ on $\mathrm{Au}$ foil was conducted in an S-rich atmosphere, at $600^{\circ} \mathrm{C}$, using CVD to evap- orate the $S$ atoms [Fig. 1(a)]. To study the effect of S-intercalation on $\mathrm{WS}_{2}$, samples were characterized using Raman spectroscopy. While the confocal Raman mapping image of as-grown $\mathrm{WS}_{2}$ samples showed the solid intensity at the triangular $\mathrm{WS}_{2}$ grain edges [Fig. 1(b)], strong Raman intensities were detected at both the inner and edge regions of the $\mathrm{WS}_{2}$ grains after S-intercalation [Fig. 1(c)]. The representative center region Raman spectra [I and II in Fig. 1(d)] showed significant Raman intensity enhancement, whereas only marginal change was observed at the edge regions [III and IV in Fig. 1(e)]. Such strong Raman intensity was attributed to the relaxed interfacial interaction between $\mathrm{WS}_{2}$ and the $\mathrm{Au}$ surface. We noted that the optimized intercalation temperature ranged from 500 to $600^{\circ} \mathrm{C}$ (not shown here). $\mathrm{WS}_{2}$ grains were found to be damaged at $700{ }^{\circ} \mathrm{C}$, whereas the intercalation was found to be inefficient at $400{ }^{\circ} \mathrm{C}$. We also found that the evaporation of $\mathrm{S}$ powders was more effective than $\mathrm{H}_{2} \mathrm{~S}$ or $\left(\mathrm{NH}_{4}\right)_{2} \mathrm{~S}$ (not shown here).

To evaluate the relaxed interfacial interaction, the interfacial distance was measured directly, using cross-sectional, annular dark-field, scanning transmission electron microscopy (ADF-STEM). In the crosssectional ADF-STEM image, we were able to distinguish the $\mathrm{WS}_{2}$ layer from the Au surface of as-grown $\mathrm{WS}_{2}$ quite clearly, as shown in Fig. 2(a). After S-intercalation, the interfacial distance had increased, from 0.24 to $0.44 \mathrm{~nm}$ [Fig. 2(b)]. This distance was further confirmed using the energy-dispersive X-ray spectroscopy (EDX) mapping technique. 
(a)

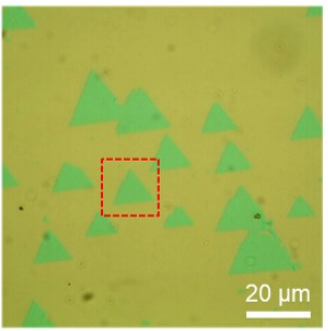

(e)

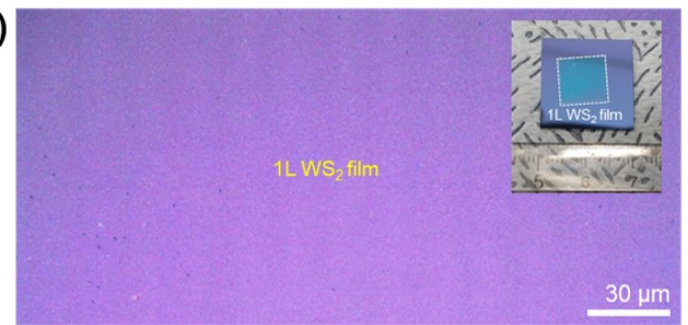

(b)

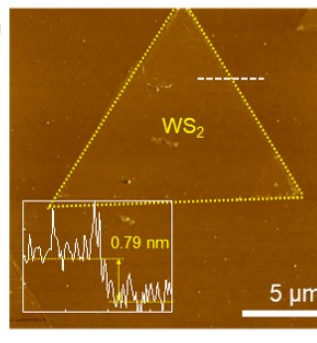

(c)

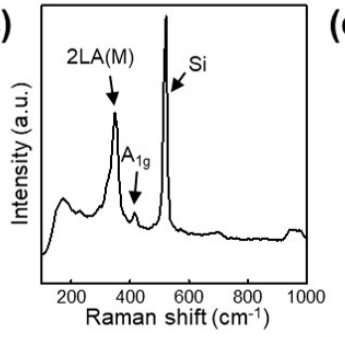

(d)

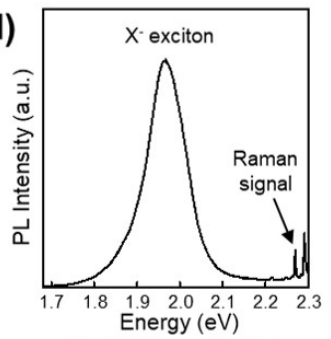

(f)

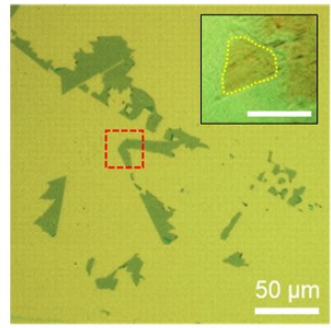

(g)

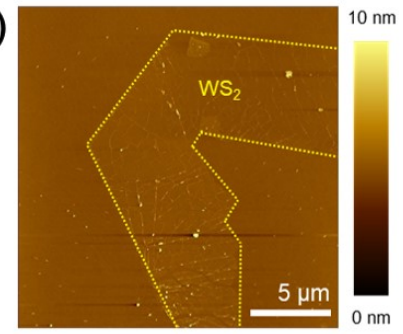

Figure 4. (a) and (b) Optical and AFM topography images of transferred WS 2 grains, after S-intercalation (AFM image was obtained from the red-dashed box, and the inset shows a height profile along the white-dashed line). (c) and (d) Raman and photoluminescence spectra, respectively, for transferred WS 2 . (e) Optical image of transferred, cm-scale monolayer $W_{2}$ film, with photograph inset. (f) Optical and (g) AFM topography images of transferred WS 2 grains without S-intercalation. The

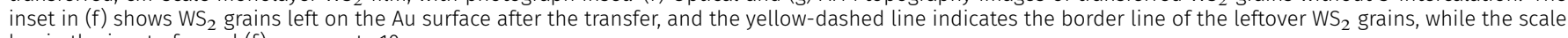
bar in the inset of panel (f) represents $10 \mu \mathrm{m}$.
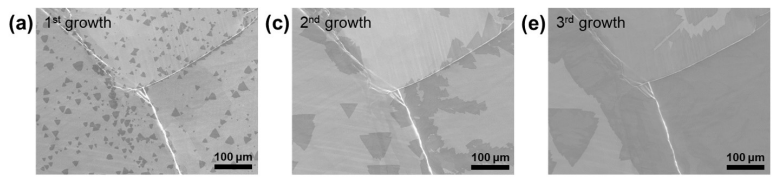

(b) After transfer \& cleaning
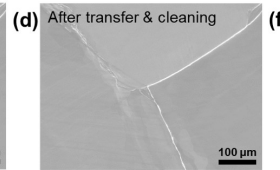

(f) After transfer \& cleaning

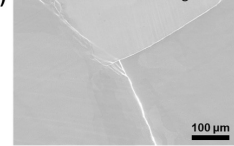

Figure 5. SEM images of $\mathrm{WS}_{2}$ after the (a) first, (c) second, and (e) third growth processes conducted using the same Au substrate. (b), (d), and (f) are SEM images of the Au substrate after electrochemical transfer and cleaning processes.

ADF-STEM and corresponding EDX mapping images of $\mathrm{WS}_{2}$ samples before and after S-intercalation showed the presence of $\mathrm{W}$ and Au elements [Figs. 2(c)-(f)]. We found it difficult to identify the presence of elemental $\mathrm{S}$ in the EDX mapping imagery, due to the $\mathrm{Au} \mathrm{M} \alpha$ and $\mathrm{S}$ $\mathrm{K} \alpha$ energy overlaps near $2.2 \mathrm{eV}$. We did find, however, that the EDX line profiles along the white arrows in Figs. 2(e) and 2(f) confirmed that the interlayer distances between $\mathrm{W}$ and $\mathrm{Au}$ atoms were similar to those directly measured in the cross-sectional ADF-STEM images.

To investigate the electronic structure modulation achieved by the $\mathrm{S}$-intercalation, samples were further characterized using KPFM. The KPFM image and its line profile at the center region of a $\mathrm{WS}_{2}$ grain after S-intercalation, yielded a contact potential difference $(\triangle \mathrm{VCPD})$ that was lower, by $76 \mathrm{mV}$, than that of the Au surface (Figs. 3(c) and $3(\mathrm{~d})$ ), whereas $\triangle \mathrm{VCPD}$ was higher, by $32 \mathrm{mV}$, in as-grown $\mathrm{WS}_{2}$ samples (Figs. 3(a) and 3(b)). The lower $\mathrm{WS}_{2} \Delta \mathrm{VCPD}$ value indicated a higher work function for $\mathrm{WS}_{2}$ than that seen for $\mathrm{Au}$.

$\mathrm{A} \mathrm{WS}_{2} / \mathrm{Au}$ energy band diagram has been presented, as Fig. 3(e), to explain the $\mathrm{WS}_{2}$ electronic structure modification-achieved by Sintercalation-which we observed in the KPFM results. Work functions of $4.82 \mathrm{eV}\left(\right.$ for $\mathrm{Au}$ ) and $4.9 \mathrm{eV}$ (for $\mathrm{WS}_{2}$ ) were selected from the previous literatures $[24,25]$. At the $\mathrm{WS}_{2}$ contact with $\mathrm{Au}$, in an as-grown sample, electrons were preferentially transferred from $\mathrm{Au}$ to $\mathrm{WS}_{2}$, due to the lower Au work function, with the result that n-doping into $\mathrm{WS}_{2}$ occurred. After S-intercalation, however, electrons could not be transferred to $\mathrm{WS}_{2}$, due to the increased interfacial distance. It is acknowledged that the electronic structure of intercalated S layer
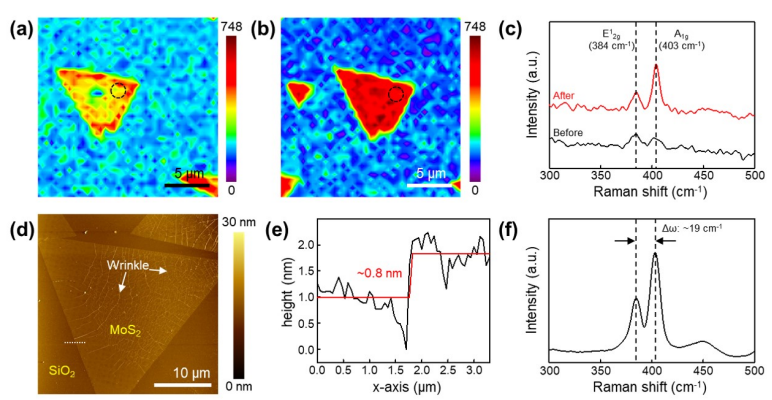

Figure 6. Confocal Raman intensity mapping images of $\mathrm{MoS}_{2}$ grains (a) before, and (b) after S-intercalation. (c) Raman spectra for $\mathrm{MoS}_{2}$ extracted from the blackdashed circles in (a) and (b). (d) AFM topography image of transferred $\mathrm{MoS}_{2}$ grains. (e) Height profile along the white-dashed line in (d). (f) Raman spectrum for a transferred $\mathrm{MoS}_{2}$ grains.

has not been revealed so far. Nevertheless, our de-doping hypothesis was supported by the KPFM results.

Finally, electrochemical bubbling transfer was conducted, after Sintercalation. The optical and corresponding AFM images of $\mathrm{WS}_{2}$ grains transferred onto the $\mathrm{SiO}_{2} / \mathrm{Si}$ substrate show the complete transfer of $\mathrm{WS}_{2}$ grains over the whole region [Figs. 4(a) and 4(b)], regardless of growth conditions. The characteristic Raman phonon mode and strong negative trion $\left(\mathrm{X}^{-}\right.$, at $\left.\sim 1.96 \mathrm{eV}\right)$ emissions can be seen clearly in Figs. 4(c) and 4(d) [18]. Furthermore, $1 \times 1 \mathrm{~cm}^{2}$-sized WS films were successfully transferred onto a $\mathrm{SiO}_{2}$ surface (Fig. 4(e) and inset). Interestingly, only edge regions of triangular $\mathrm{WS}_{2}$ grains were transferred without S-intercalation, as seen in Figs. 4(f) and 4(g), with the center regions remaining on the Au surface, as seen in the inset of Fig. 4(f). These results strongly supported the concept that weakening the interfacial interaction was a crucial factor in achieving a high transfer yield. Furthermore, the confocal Raman mapping images in Figs. 1(b) and 1(c) can be used as one of important indicators in determining the transfer yield - that is, the regions where the $\mathrm{WS}_{2}$ Raman intensity was strong, were easily delaminated from the Au surface. In addition, after the $\mathrm{WS}_{2}$ had been transferred, the Au substrate was reused for further growth processes, as shown in Fig. 5. The surface morphology remained virtually identical to a pristine Au surface and was readily available for further $\mathrm{WS}_{2}$ growth. 
(a)
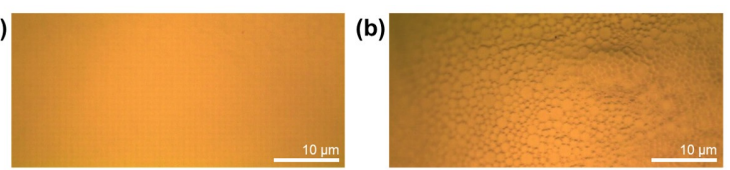

(c)

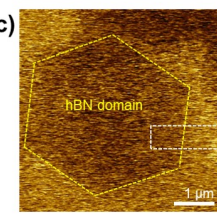

(f)

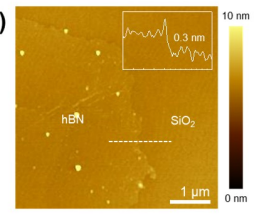

(e)

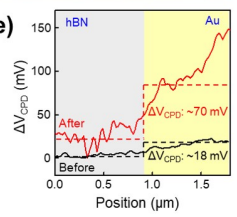

(g)

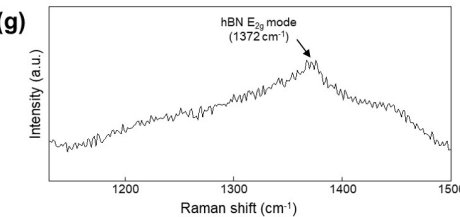

Figure 7. Optical images of (a) as-grown, and (b) S-intercalated hBN grains, on an Au substrate. KPFM potential images of hBN grains (c) before and (d) after S intercalation. (e) Potential profiles along the white-dashed boxes in (c) and (d) (f) AFM topography image and (g) Raman spectrum respectively, of monolayer hBN grains after their transfer onto a $\mathrm{SiO}_{2} / \mathrm{Si}$ substrate. The inset of (f) shows a height profile extracted from the white-dashed line.

To demonstrate the universal transfer of $2 \mathrm{D}$ materials grown on Au surfaces, molybdenum disulfide $\left(\mathrm{MoS}_{2}\right)$ and hBN were also transferred, after $S$-intercalation. The enhancements of $E^{1}{ }_{2 g}$ and $A_{1 g}$ phonon modes for $\mathrm{MoS}_{2}$ after S-intercalation can be seen quite clearly in the $\mathrm{MoS}_{2}$ confocal Raman mapping images shown in Figs. 6(a)-(c), in consistent with the change of Raman intensity seen for $\mathrm{WS}_{2}$. AFM image of $\mathrm{MoS}_{2}$ flakes transferred onto a $\mathrm{SiO}_{2} / \mathrm{Si}$ substrate shows the wrinkles on the $\mathrm{MoS}_{2}$ flakes, demonstrating successful $\mathrm{MoS}_{2}$ transfer, as seen in Fig. 6(d). Extracted height profiles, combined with the phonon energy differences ( $\omega$ ) of $\sim 19 \mathrm{~cm}^{-1}$ in the Raman spectrum, were further evidence of the successful transfer of a $\mathrm{MoS}_{2}$ flake monolayer [Figs. 6(e) and 6(f)] [12].

For hBN, the optical image of as-grown $\mathrm{hBN}$ on $\mathrm{Au}$ foil shows no noticeable hBN flakes, while they are precisely visible after the $S$ intercalation process [Figs. 7(a) and 7(b)]. The surface potential difference between as-grown $\mathrm{hBN}$ flakes and $\mathrm{Au}$ was found to be small $(\sim 18 \mathrm{mV})$ in a pristine sample. This became larger $(\sim 70 \mathrm{mV})$ after S-intercalation [Figs. 7(c) and 7(e)]. This variation might be ascribed to the change in charge transfer behavior between $\mathrm{hBN}$ and the $\mathrm{Au}$ surface, brought about by the presence of an interfacial S layer. After a transfer of hBN flakes, the monolayer thickness of $\mathrm{hBN}$ and its phonon mode of $E_{2 \mathrm{~g}}$ near $1372 \mathrm{~cm}^{-1}$ were characterized, with the results shown in Figs. 7(f) and 7(g) [13].

\section{Conclusions}

In summary, we have successfully developed a method for the universal transfer of $2 \mathrm{D}$ materials grown on Au foil, using S-intercalation at the interface between the $2 \mathrm{D}$ materials and the Au surface. The S-intercalation extended the interfacial distance, as was conclusively confirmed using cross-sectional TEM. Raman spectroscopy, and KPFM provided further evidence that the $S$ layer blocked charge transfers between the 2D materials and the Au surface. This releasing interaction

enabled the successful transfer of $\mathrm{cm}$-scale, monolayer $\mathrm{WS}_{2}$ film. We anticipate that our novel transfer method will contribute to the use of SC 2D monolayers grown on Au foil in industrial applications.

\section{Acknowledgements}

This research was supported by the Institute for Basic Science (IBSR011-D1), and by the Basic Research Program of the National Research Foundation of Korea (NRF), funded by the Ministry of Science, ICT \& Future Planning (Grant Nos 2018R1A2B2002302, 2020R1A2C1006207, and 2020R1A4A3079710). H. Y. J. acknowledges support from the Creative Materials Discovery Program through the National Research Foundation of Korea (NRF-2016M3D1A1900035). S. M. K. acknowledges support by Samsung Research Funding Incubation Center of Samsung Electronics under Project Number SRFC-MA1901-04.

\section{References}

[1] N. M. R. Peres, A. H. Castro Neto, and F. Guinea, Phys. Rev. B. 73, 241403 (2006).

[2] J. McClain and J. Schrier, J. Phys. Chem. C. 114, 14332 (2010).

[3] T. T. Tran, K. Bray, M. J. Ford, M. Toth, and I. Aharonovich, Nat. Nanotech. 11, 37 (2016).

[4] Y. Jin, M. K. Joo, B. H. Moon, H. Kim, S. H. Lee, H. Y. Jeong, and Y. H. Lee, Commun. Phys. 3, 189 (2020).

[5] M. I. Vasilevskiy, D. G. Santiago-Perez, C. Trallero-Giner, N. M. R. Peres, and A. Kavokin, Phys. Rev. B. 92, 245435 (2015)

[6] D. Manh-Ha, Y. Jin, K. C. Tuan, M. K. Joo, and Y. H. Lee, Adv. Mater. 31, 1900154 (2019).

[7] D. Fu et al., J. Am. Chem. Soc. 139, 9392 (2017).

[8] L. K. Tan, B. Liu, J. H. Teng, S. Guo, H. Y. Low, and K. P. Loh, Nanoscale 6, 10584 (2014).

[9] F. Tumino, C. S. Casari, M. Passoni, V. Russo, and A. L. Bassi, Nanoscale Advances 1, 643 (2019).

[10] J. Y. Chen et al., J. Am. Chem. Soc. 139, 1073 (2017).

[11] Y. Zhang et al., ACS Nano 8, 8617 (2014).

[12] K. Kang, S. E. Xie, L. J. Huang, Y. M. Han, P. Y. Huang, K. F. Mak, C. J. Kim, D. Muller, and J. Park, Nature 520, 656 (2015).

[13] J. H. Park et al., ACS Nano 8, 8520 (2014).

[14] X. S. Li et al., Science 324, 1312 (2009).

[15] Y. Gao et al., Adv. Mater. 29, 1700990 (2017).

[16] J. S. Lee et al., Science 362, 817 (2018).

[17] T. A. Chen et al., Nature 579, 219 (2020).

[18] S. J. Yun et al., ACS Nano 9, 5510 (2015).

[19] T. H. Ly, D. J. Perello, J. Zhao, Q. M. Deng, H. Kim, G. H. Han, S. H. Chae, H. Y. Jeong, and Y. H. Lee, Nat. Commun. 7, 10426 (2016).

[20] S. H. Choi et al., arXiv:2010.10097, (2020).

[21] S. M. Kim, A. Hsu, Y. H. Lee, M. Dresselhaus, T. Palacios, K. K. Kim, and J. Kong, Nanotechnology 24, 365602 (2013).

[22] S. H. Choi, C. S. Oh, S. Boandoh, W. Yang, S. M. Kim, and K. K. Kim, Appl. Sci. Converg. Tec. 28, 60 (2019).

[23] Y. Chen et al., ACS Nano 12, 2569 (2018).

[24] J. H. Cha, S. J. Choi, S. Yu, and I. D. Kim, J. Mater. Chem. A. 5, 8725 (2017).

[25] V. Panchal, R. Pearce, R. Yakimova, A. Tzalenchuk, and O. Kazakova, Sci. Rep. 3, 2597 (2013). 